\title{
Giant magnetocaloric effect of thin Ho films
}

F. C. Medeiros Filho, V. D. Mello, A. L. Dantas, F. H. S. Sales, and A. S. Carriço

Citation: Journal of Applied Physics 109, 07 A914 (2011);

View online: https://doi.org/10.1063/1.3549566

View Table of Contents: http://aip.scitation.org/toc/jap/109/7

Published by the American Institute of Physics

\section{Articles you may be interested in}

Magnetocaloric effect in nanoscale thin films and heterostructures

Journal of Vacuum Science \& Technology A: Vacuum, Surfaces, and Films 32, 040802 (2014);

$10.1116 / 1.4882858$

Magnetocaloric effect in Gd/W thin film heterostructures

Journal of Applied Physics 107, 09A903 (2010); 10.1063/1.3335515

Magnetocaloric properties of doped lanthanum manganite films

Journal of Applied Physics 79, 373 (1996); 10.1063/1.360840

Correlation between structural parameters and the magnetocaloric effect in epitaxial $\mathrm{La}_{0.8} \mathrm{Ca}_{0.2} \mathrm{MnO}_{3} / \mathrm{LaAlO}_{3}$ thin film

Journal of Applied Physics 113, 063508 (2013); 10.1063/1.4790876

Impact of interfacial magnetism on magnetocaloric properties of thin film heterostructures

Journal of Applied Physics 109, 063905 (2011); 10.1063/1.3555101

Magnetocaloric effect in Ni-Mn-Ga thin films under concurrent magnetostructural and Curie transitions Journal of Applied Physics 110, 013910 (2011); 10.1063/1.3602088

\section{Scilight}

Sharp, quick summaries illuminating the latest physics research

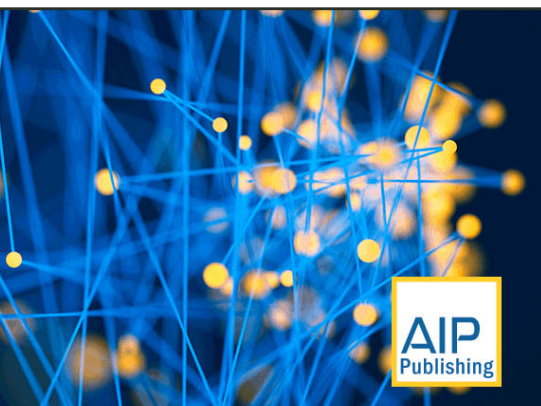




\title{
Giant magnetocaloric effect of thin Ho films
}

\author{
F. C. Medeiros Filho, ${ }^{1,2}$ V. D. Mello, ${ }^{1,2}$ A. L. Dantas, ${ }^{1}$ F. H. S. Sales,,${ }^{2,3}$ and A. S. Carriço ${ }^{2, a)}$ \\ ${ }^{1}$ Department of Physics, UERN, Mossoró, RN, Brazil 59610-210 \\ ${ }^{2}$ Department of Physics, UFRN, Natal, RN, Brazil 59072-970 \\ ${ }^{3}$ Department of Physics, IFMA, São Luis, MA, Brazil 65030-005
}

(Presented 15 November 2010; received 24 September 2010; accepted 10 November 2010; published online 24 March 2011)

\begin{abstract}
We report a theoretical study of the impact of finite size on the magnetocaloric effect (MCE) of thin Ho films. For strong external field strengths, the adiabatic temperature change $\Delta T_{a d}$ is comparable to the value found in bulk Ho, reaching about $12 \mathrm{~K}$ for an external field strength change $\Delta H=50 \mathrm{kOe}$. For thicknesses below the helix period, there is a large enhancement. In this thickness range, the helical state does not form, leading to a giant MCE reaching $\Delta T / \Delta H=6.5 \mathrm{~K} / \mathrm{T}$ for $\Delta H=2 \mathrm{kOe}$. (C) 2011 American Institute of Physics. [doi:10.1063/1.3549566]
\end{abstract}

Considerable research effort has been dedicated to investigating the impact of confinement on the equilibrium phases and excitations of magnetic systems of a size comparable to fundamental magnetic lengths. Confinement effects are particularly interesting in magnetic systems in which the leading bulk magnetic phases consist of patterns made of a periodical repetition of units containing a finite number of spins. This is the case with the helical state of rare earth metals, and there are interesting reports revealing strong finite size effects on films with thicknesses below a few helix periods.

Thin Dy films ${ }^{1}$ and thin film Gd/Dy/Gd and $\mathrm{Fe} / \mathrm{Dy} / \mathrm{Fe}$ trilayers $^{2}$ display large thermal hysteresis, induced by the low temperature nucleation of an alternate helicity phase, locking surface spins to the external field direction. New magnetic phases of thin Dy films have been reported, ${ }^{3,4}$ as well as a giant magnetocaloric effect (MCE) for ultrathin Dy films for external field strengths of a few kOe. ${ }^{5}$

In this article, we investigate the MCE of Ho films with thicknesses less than the helix period. Our results indicate that the helical state does not form in films with a thickness below six atomic layers. As a result, the efficiency of the temperature change by adiabatic demagnetization, as measured from the ratio $\Delta T / \Delta H$, reaches values ten times larger than the corresponding bulk values for external fields of a few kOe.

A strong thickness dependence of the Neel temperature of Ho thin films has been recently reported, indicating that the helical state does not form in Ho films with a thickness below eight atomic layers. ${ }^{6}$ Holmium orders in a basal plane helical phase in the temperature interval from $20 \mathrm{~K}$ to $132 \mathrm{~K}$. The helix turn angle $\delta \varphi$ varies between $30^{\circ}$ and $50^{\circ}$. Therefore, the helix period corresponds to nearly nine atomic layers, and one might expect strong surface effects for films in this thickness range.

Details of the helimagnetic state and of confinement and surface effects may be modeled by the competition between first neighbor ferromagnetic exchange interaction and second neighbor antiferromagnetic exchange interaction. ${ }^{1,7}$

Two features are worth noticing. First, the turn angle in the near surface region is likely to be smaller. Near the sur-

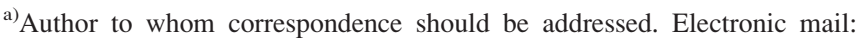
ascarrico@gmail.com.
}

face, the reduction of the antiferromagnetic next-nearestneighbor exchange energy is larger than the reduction of the ferromagnetic nearest neighbor exchange energy. This leads to changes in the exchange energy balance, favoring a more ferromagnetic arrangement for spins in the surface layers.

Second, although the spins of only the first two surface atomic layers have a reduced coordination number, surface effects are not restricted to the surface layers. The impact of surface effects depends on the way the effective local field relaxes toward the bulk pattern in the middle of the film. Thus, confinement may affect the whole film, inhibiting the formation of the helical state below a threshold value for the film's thickness.

There is currently renewed interest in new materials for magnetocaloric applications after the pioneering work revealing the giant MCE of $\mathrm{Gd}_{5}\left(\mathrm{Si}_{2} \mathrm{Ge}_{2}\right) .{ }^{8,9}$ The motivation for studies in Gd-based systems stems from the potential of solid magnetic systems as a substitute for traditional gas compression technologies in room temperature refrigeration. ${ }^{9}$

Materials based on Dy and Ho, which have the largest magnetic moment among the rare earth elements, are the best choice for low temperature MCE applications. ${ }^{9}$ Measurements of the MCE in polycrystalline $\mathrm{Ho}^{10}$ revealed an adiabatic temperature change, at the Neel temperature, of $\Delta T=4.5 \mathrm{~K}$ for an external field variation of $\Delta H=60 \mathrm{kOe}$, and $\Delta T=6.1 \mathrm{~K}$ for $\Delta H=70 \mathrm{kOe}$. These values of the Holmium adiabatic temperature change under fields on the order of a few Tesla are smaller than those reported for Dysprosium under fields of the same magnitude, ${ }^{11}$ possibly because the Holmium samples were polycrystalline.

Our present results suggest that much larger adiabatic temperature changes are expected for crystalline samples under external fields above 2T. Furthermore, with ultrathin films and an external field strength of a few kOe, one may achieve a large MCE efficiency. For instance, for an Ho film with five atomic layers, we have found an adiabatic temperature change of $\Delta T=1.3 \mathrm{~K}$, at a temperature of $121 \mathrm{~K}$, for an external field of $2 \mathrm{kOe}$. The corresponding value for bulk Ho is $\Delta T=0.08 \mathrm{~K}$ at the Neel temperature. Therefore, the bulk MCE is one order of magnitude smaller than the thin film MCE. 
We investigate a $c$-axis thin film consisting of a stack of atomic layers with equivalent spins, infinitely extended in the $x-y$ directions. The spins in each monolayer are exchange coupled with the spins in the first and second neighbor monolayers. The anisotropy is uniform throughout the film, and the near surface spins have a reduced exchange energy. The magnetic Hamiltonian is given by:

$$
\begin{aligned}
H= & J_{1}(g-1)^{2} \sum_{n=1}^{N-1} \vec{J}(n) \cdot \vec{J}(n+1) \\
& +J_{2}(g-1)^{2} \sum_{n=1}^{N-2} \vec{J}(n) \cdot \vec{J}(n+2) \\
& +\sum_{n=1}^{N}\left[K_{6}^{6} \cos \left(6 \varphi_{n}\right)-g \mu_{B} \vec{J}(n) \cdot \vec{H}\right] .
\end{aligned}
$$

$J_{1}$ and $J_{2}$ are the exchange energy between spins in the nearest and next nearest monolayers, and $\vec{J}(n)$ denotes the total angular momentum per ion in the $n^{\text {th }}$ monolayer. $K_{6}^{6}$ describes the hexagonal anisotropy, and the last term is the Zeeman energy, with the external field $\vec{H}$ along one easyaxis direction in the basal plane. ${ }^{3}$

We use the Ho bulk energy parameters ${ }^{12} J=8$, $J_{1}=47 k_{B}$, and $J_{2}=-J_{1} / 4 \cos \phi(T)$, where $\phi(T)$ is the temperature dependent helix turn angle. $g=5 / 4$ is the Lande factor, corresponding to a saturation magnetic moment per atom of $10.6 \mu_{B}$. For the temperature dependence of the anisotropy, $K_{6}^{6}(T)$, and of the turn angle, $\phi(T)$, we use the values reported for bulk Ho. ${ }^{12,13}$

The equilibrium magnetic pattern for each value of the external field at a given temperature is obtained using a selfconsistent local field model that incorporates the surface modifications in the exchange field and allows for the mutual adjustment of the thermal average values $[\langle J(n)\rangle ; n=1 ; \ldots N]$ and the orientation of the spins in each layer $\left(\left\langle\phi_{n}\right\rangle\right.$; $n=1 ; \ldots N){ }^{1-5}$

The adiabatic temperature change is calculated using:

$$
\Delta T_{a d}(T, H)=-\int_{H_{i}}^{H_{f}}\left(\frac{T}{C(T, H)}\right)_{H}\left(\frac{\partial M(T, H)}{\partial T}\right)_{H} d H
$$

where $C(T, H)$ is the total specific heat and includes both magnetic and elastic (lattice) contributions, and $[\partial M(T, H) /$ $\partial T]_{H}$ is the temperature derivative of the magnetization along the external field direction. ${ }^{9}$

One may anticipate the impact of finite size effects by making a comparison with the case of ferromagnetic materials. The main point is that by making the Ho film thin enough, one inhibits the formation of the helical phase, leading to an increase in the value of the MCE.

MCE originates in the variations of the magnetic entropy upon changes in the external magnetic field. As a result, the MCE is larger for temperature values for which there is a relevant impact of the external field on the magnetic order. For ferromagnetic materials, this amounts to the region near the Curie temperature, where the exchange and anisotropy fields may become comparable to or smaller than the external field.
For helimagnetic materials, near the Neel point, rather small variations in the external field strength lead to transitions from the helimagnetic phase to the fan phase and to the ferromagnetic phase (aligned with the external field). Within the helimagnetic phase, one may find anomalous MCE, because there is a fraction of the spins in the helical structure, which is aligned at large angles to the external field. For these spins, reduction of the external field leads to a decrease in the magnetic entropy. Also, at points near the border between the helical and fan phases, by increasing the temperature one gains more alignment with $H[(\partial M(T, H) /$ $\left.\partial T)_{H}>0\right]$.

As can be seen from Eq. (2), there is a relevant contribution to $\Delta T$ from small field values, because in this case the derivative of the magnetization is larger. Thus, for any temperature, the low field end of the interval of integration leads to a negative MCE. This originates the negative MCE of helimagnetic materials for fields on the order of a few kOe. For Dy, for instance, only for fields above $2 \mathrm{~T}$ do the MCE curves assume the caretlike shape of the normal MCE. ${ }^{11}$

In the following results we have chosen $H_{i}=0$, so that for normal MCE the adiabatic temperature change $\left(\Delta T_{a d}\right)$ is positive and corresponds to the process of heating the sample by switching on the external field adiabatically.

The adiabatic temperature change of a five monolayer Ho film, shown in Fig. 1, displays the caretlike shape typical of ferromagnetic materials near the Curie temperature. For an external field of $2 \mathrm{kOe}$, the temperature increases by around $1.3 \mathrm{~K}$. The peak in $\Delta T_{a d}(T)$ occurs at a temperature of $121 \mathrm{~K}$. The corresponding $\Delta T_{a d}(T)$ curve for bulk Ho displays the anomalous MCE, typical of the helimagnetic phase at small external field strength values. The peak at the Neel temperature is rather small $\left[\Delta T_{a d}\left(T_{N}\right) \approx 0.08 \mathrm{~K}\right]$. The magnitude of the MCE of the thin Ho film is one order of magnitude larger. The curve for the bulk was calculated by imposing cyclic boundary conditions on a slab with nine monolayers. We have also checked other cyclic boundary conditions (for $N$ around nine, as is appropriate for the temperature range near the Neel point) and found similar results.

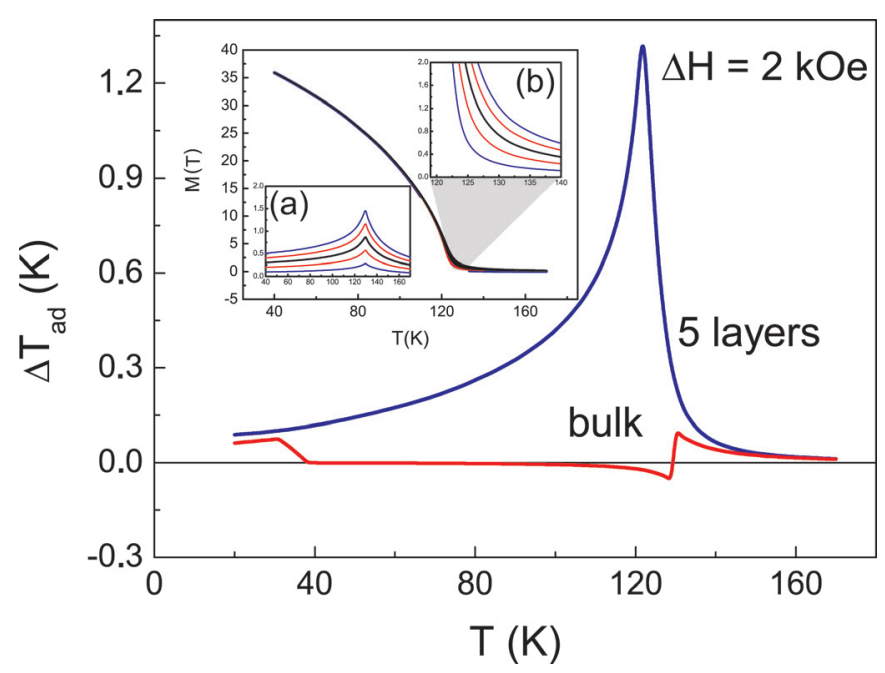

FIG. 1. (Color online) Adiabatic temperature change for a 5 monolayer Ho film and for bulk Ho, $\Delta H=2 \mathrm{kOe}$. Notice that the peak value of $\Delta T$ for the thin film is more than ten times larger than the corresponding bulk value. 


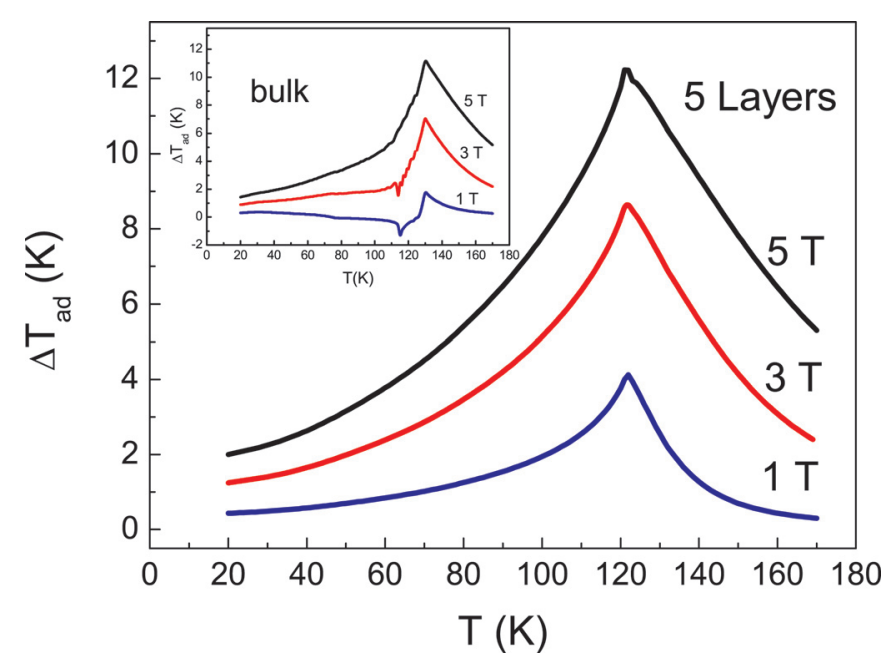

FIG. 2. (Color online) Adiabatic temperature change for a 5 monolayer Ho film and for bulk Ho (inset). The numbers by the curves indicate the values of $\Delta H$.

In the inset of Fig. 1 we show the $M(T)$ curves for the five monolayer film for external field values of $0.2 \mathrm{kOe}, 0.4$ $\mathrm{kOe}, 0.6 \mathrm{kOe}, 0.8 \mathrm{kOe}$, and $1 \mathrm{kOe}$ and the high temperature details of [Fig. 1(a)] bulk $M(T)$ curves and [Fig. 1(b)] the thin film $M(T)$ curves. The change in slope from positive below the Neel point to negative above it, as shown in Fig. 1(a), explains the anomalous MCE of bulk Ho. The

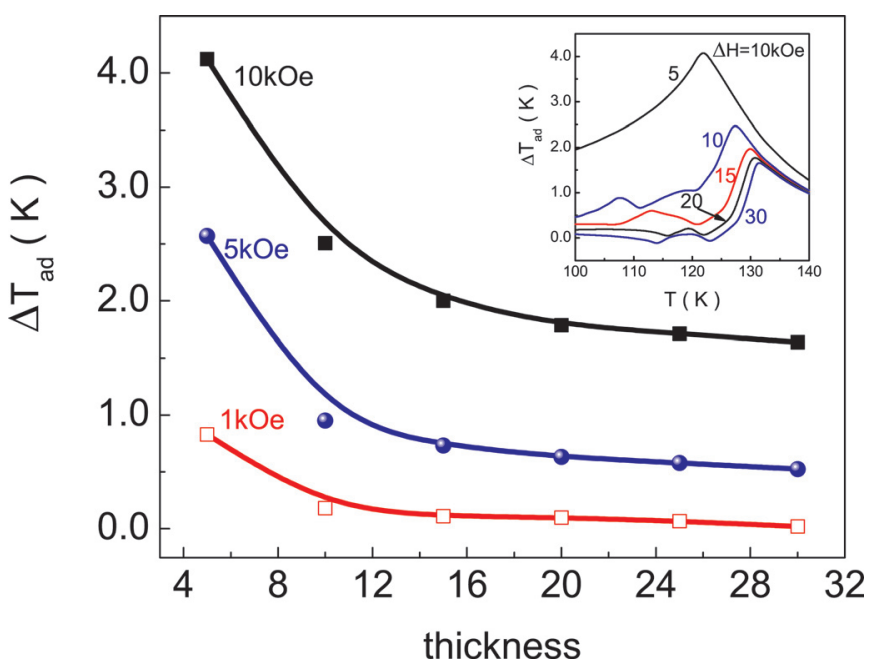

FIG. 3. (Color online) Thickness dependence of the value of the adiabatic temperature change at the Neel temperature. The numbers by the curves indicate the values of the external field. In the inset we show the $\Delta T_{a d}(T)$ curves for an external field value of $10 \mathrm{kOe}$. The number of layers is indicated in the numbers by the curves.
$M(T)$ curves in Fig. 1(b) with large negative slopes are the cause of the large MCE of the thin Ho film.

The enhancement of the MCE for large field strengths, as shown in Fig. 2, is not relevant, except for $\Delta H=10$ kOe. Also, as is shown in Fig. 3, the MCE efficiency increases as the thickness is reduced. Furthermore, the finite size enhancement of the MCE is larger for a small field strength.

In summary, we have shown that the MCE efficiency of Ho is significantly enhanced by finite size effects in ultrathin films. Confinement inhibits the formation of the helical state. Ultrathin Ho films are ferromagnetic in the whole temperature range from the Curie temperature to the Neel temperature. As a result, the adiabatic temperature change may be much larger than the corresponding value for bulk Holmium. For external field strengths on the order of a few kOe, the thin film MCE is an order of magnitude larger than the value found for bulk Holmium.

The present results suggest that thin films or Ho nanoparticles might be a promising component of MCE devices for low temperature applications.

The authors acknowledge support from CNPq, CAPES, FAPERN, and FAPEMA. The work of A. S. Carrico was supported by CNPq Grant 350773. The work of A. L. Dantas was supported by CNPq Grant 309676.

${ }^{1}$ A. L. Dantas, R. E. Camley, and A. S. Carriço, IEEE Trans. Magn. 42, 2942 (2006)

${ }^{2}$ A. L. Dantas, R. E. Camley, and A. S. Carriço, Phys. Rev. B 75, 094436 (2007)

${ }^{3}$ V. D. Mello, C. V. Chianca, A. L. Dantas, and A. S. Carriço, Phys. Rev. B 67, 012401 (2003)

${ }^{4}$ V. D. Mello and A. S. Carriço, Surf. Sci. 482, 960 (2001).

${ }^{5}$ V. D. Mello, A. L. Dantas, and A. S. Carriço, Solid State Commun. 140, 447 (2006).

${ }^{6}$ E. Weschke, H. Ott, E. Schierle, C. Schüßler-Langeheine, D. V. Vyalikh, G. Kaindl, V. Leiner, M. Ay, T. Schmitte, H. Zabel, and P. J. Jensen, Phys. Rev. Lett. 93, 157204 (2004).

${ }^{7}$ R. E. Camley, J. Kwo, M. Hong, and C. L. Chien, Phys. Rev. Lett. 64, 2703 (1990).

${ }^{8}$ V. K. Pecharsky and K. A. Gschneidner, Phys. Rev. Lett. 78, 4494 (1997).

${ }^{9}$ K. A. Gschneidner, Jr., V. K. Pecharsky, and A. O. Tsokol, Rep. Prog. Phys. 68, 1479 (2005).

${ }^{10}$ S. A. Nikitin, A. S. Andreenko, A. M. Tishin, A. M. Arkharov, and A. A. Zherdev, Phys. Met. Metallogr. 60, 56 (1985).

${ }^{11}$ S. M. Benford, J. Appl. Phys. 50, 1868 (1977); A. S. Chernyshov, A. O. Tsokol, A. M. Tishin, K. A. Gschneidner, Jr., and V. K. Pecharsky, Phys. Rev. B 71, 184410 (2005).

${ }^{12}$ S. Legvold, Rare Earth Metals and Alloys, (North-Holland, Amsterdam, 1980); A. M. Ventery and P. V. Plessisz, J. Phys. Condens. Matter 9, 5167 (1997).

${ }^{13}$ B. Coqblin, The Electronic Structure of Rare Earth Metals and Alloys: The Magnetic Heavy Rare-Earths (Academic, New York, 1977). 